\title{
PROPERTIES OF FIXED-POINT SETS OF NONEXPANSIVE MAPPINGS IN BANACH SPACES
}

\author{
BY \\ RONALD E. BRUCK, JR.
}

\begin{abstract}
Let $C$ be a closed convex subset of the Banach space $X$. A subset $F$ of $C$ is called a nonexpansive retract of $C$ if either $F=\varnothing$ or there exists a retraction of $C$ onto $F$ which is a nonexpansive mapping. The main theorem of this paper is that if $T: C \rightarrow C$ is nonexpansive and satisfies a conditional fixed point property, then the fixed-point set of $T$ is a nonexpansive retract of $C$. This result is used to generalize a theorem of Belluce and Kirk on the existence of a common fixed point of a finite family of commuting nonexpansive mappings.
\end{abstract}

Introduction. In this paper, $X$ always denotes a Banach space (either real or complex) and $C$ a nonempty, closed and convex subset of $X$.

Most published results on nonexpansive mappings have centered on existence theorems for fixed points of nonexpansive $T: C \rightarrow X$. In this paper we initiate the study of the structure of the fixed-point set $F(T)=\{x: T x=x\}$. In this connection it is useful to assume that a conditional fixed point property is satisfied:

Either $T$ has no fixed points, or $T$ has a fixed

point in every nonempty bounded closed convex

set that $T$ leaves invariant.

It is obvious that existence theorems serve to define classes of nonexpansive mappings which satisfy (CFP). However, (CFP) holds even in contexts where no existence theorem can be hoped for. For example (Theorem 4), if $C$ is locally weakly compact and $X$ is strictly convex, then every nonexpansive $T: C \rightarrow X$ satisfies (CFP).

Our principal structure result is Theorem 2: if $C$ is locally weakly compact, $T: C \rightarrow C$ is nonexpansive, and $T$ satisfies (CFP), then $F(T)$ is a nonexpansive retract of $C$. Therefore our approach is to study the structure of $F(T)$ by studying nonexpansive retracts of $C$.

Although we are not primarily interested in existence results, our main structure theorem does permit us to prove an existence result (Theorem 7) under more general hypotheses than before, by a more transparent argument.

Received by the editors November 13, 1970 and, in revised form, May 19, 1972.

AMS (MOS) subject classifications (1970). Primary 47HIO.

Key words and phrases. Fixed-point set, metrically convex, nonexpansive mapping, nonexpansive retract, normal structure.

Copyright O 1973, American Mathematical Society 
Some of the results in this paper were announced in Bruck [4] under less general hypotheses.

1. Nonexpansive retracts. A mapping $T: C \rightarrow X$ is said to be nonexpansive if $\|T x-T y\| \leq\|x-y\|$ for all $x, y \in C$.

Our main tool is

Lemma 1. Suppose $F$ is a nonempty subset of the locally weakly compact, convex set $C$, and let $N(F)=\{f: f: C \rightarrow C$ is nonexpansive and $f x=x$ for all $x \in F\}$. Then $N(F)$ is compact in the topology of weak pointwise convergence.

Proof. Fix $x_{0} \in F$ and for $x \in C$ define

$$
C_{x}=\left\{y \in C:\left\|y-x_{0}\right\| \leq\left\|x-x_{0}\right\|\right\} \text {. }
$$

For each $x$ in $C$ and $f$ in $N(F), f(x) \in C_{x}$ since $f(C) \subset C$ and

$$
\left\|f x-x_{0}\right\|=\left\|f x-f x_{0}\right\| \leq\left\|x-x_{0}\right\| .
$$

Thus $N(F)$ is a subset of the Cartesian product $P=\prod_{x \in C} C_{x}$.

Since $C$ is convex and locally weakly compact, each $C_{x}$ is convex and weakly compact. If $C_{x}$ is given the weak topology and $P$ is given the corresponding product topology, then by Tychonoff's theorem $P$ is compact. (The topology on $P$ is that of weak pointwise convergence, i.e., a net $\left\{f_{\lambda}: \lambda \in \Lambda\right\}$ in $P$ converges to $f$ in $P$ iff for each $x$ in $C$ the net $\left\{f_{\lambda}(x): \lambda \in \Lambda\right\}$ converges weakly to $f(x)$.)

We show that $N(F)$ is closed in $P$. Suppose that $\left\{f_{\lambda}: \lambda \in \Lambda\right\}$ is a net in $N(F)$ which converges to $f$ in $P$. Then for $u$ in $F, f_{\lambda}(u) \equiv u$ so $f(u)=w-\lim _{\lambda} f_{\lambda}(u)=u$. By the weak lower semicontinuity of $\|\cdot\|$, for any $x, y$ in $C$,

$$
\|f x-f y\|=\left\|\mathrm{w}-\lim _{\lambda}\left(f_{\lambda} x-f_{\lambda} y\right)\right\| \leq \lim _{\lambda} \inf \left\|f_{\lambda} x-f_{\lambda} y\right\| \leq\|x-y\|
$$

since each $f_{\lambda}$ is nonexpansive.

We have shown that $f \in N(F)$, hence that $N(F)$ is closed in $P$. Since $P$ is compact, therefore $N(F)$ is compact. Q.E.D.

As a first application of Lemma 1, we have

Lemma 2. Suppose $F$ is a nonempty subset of $C$ and $C$ is locally weakly compact. Then there exists an $r$ in $N(F)$ such that each $f$ in $N(F)$ acts as an isometry on the range of $r$ :

$$
\|f r(x)-f r(y)\|=\|r(x)-r(y)\|
$$

for all $x, y$ in $C$.

Proof. Define an order $<$ on $N(F)$ by setting $f<g$ if $\|f x-f y\| \leq\|g x-g y\|$ for all $x, y$ in $C$ with inequality holding for at least one pair of $x, y$. As usual, $f \leq g$ means $f<g$ or $f=g$. It is readily verified that $\leq$ is a partial order on $N(F)$.

For each $f$ in $N(F)$ we define the initial segment Is $(f)$ to be $\{g \in N(F): g$ $\leq f\}$. The proof in Lemma 1 that $N(F)$ is closed in $P$ can be repeated to show that $\operatorname{Is}(f)$ is closed in $N(F)$, and hence compact. 
It follows that $(N(F), \leq)$ contains a minimal element. Indeed, by Zorn's lemma it suffices to show that whenever $\left\{g_{\lambda}: \lambda \in \Lambda\right\}$ is linearly ordered by $\leq$, then there exists $g$ in $N(F)$ with $g \leq g_{\lambda}$ for all $\lambda$. But if the $g_{\lambda}$ 's are linearly ordered by $\leq$, the family $\left\{\operatorname{Is}\left(g_{\lambda}\right): \lambda \in \Lambda\right\}$ is linearly ordered by inclusion. Since each Is $\left(g_{\lambda}\right)$ is compact and nonempty, there exists $g \in \bigcap_{\lambda} \operatorname{Is}\left(g_{\lambda}\right)$, i.e., there exists $g$ in $N(F)$ with $g \leq g_{\lambda}$ for each $\lambda$.

Hence there exists a minimal element $r$ of $N(F)$. It is readily verified that $f \circ r \in N(F)$ whenever $f \in N(F)$, and certainly

$$
\|f r(x)-f r(y)\| \leq\|r(x)-r(y)\|
$$

since $f$ is nonexpansive. If inequality holds in (1) for any pair $x, y$ in $C$ then $f r<r$, while $f r \in N(F)$, contradicting the minimality of $r$ in $N(F)$. Therefore equality holds in (1) for each $x, y$ in $C$. Q.E.D.

We shall call a subset $F$ of $C$ a nonexpansive retract of $C$ if either $F=\varnothing$ or there exists a retraction of $C$ onto $F$ which is a nonexpansive mapping. (We allow the empty set to avoid trivial cases in later theorems.) Nonexpansive retracts of $C$ are characterized as follows:

Theorem 1. Suppose $C$ is locally weakly compact and $F$ is a nonempty subset of $C$. Suppose that for each $z$ in $C$ there exists $h$ in $N(F)$ such that $h(z) \in F$. Then $F$ is a nonexpansive retract of $C$.

Proof. By Lemma 2, there exists $r$ in $N(F)$ such that each element of $N(F)$ acts as an isometry on the range of $r$. Since $r$ is already nonexpansive and fixes each. point of $F$, to show that $r$ is a nonexpansive retraction of $C$ onto $F$ it suffices to show that $r(p) \in F$ for each $p$ in $C$.

Applying the hypothesis of the theorem to the point $z=r(p)$, there exists $h$ in $N(F)$ with $h(r(p)) \in F$. Let $y=h(r(p))$. By Lemma 2,

$$
\|h(r(p))-h(r(y))\|=\|r(p)-r(y)\| .
$$

But since $y \in F$ we have $y=h(r(y))$, while $y=h(r(p))$ by definition. It follows from (2) that $r(p)=r(y)$. Finally, $r(y)=y$ since $y \in F$. We have shown that $r(p)=y$ is a point of $F$ for each point of $C$, which completes the proof that $r$ is a nonexpansive retraction of $C$ onto $F$. Q.E.D.

2. Fixed point sets as nonexpansive retracts. Recall that $T: C \rightarrow X$ is said to satisfy the conditional fixed point property (CFP) if either $T$ has no fixed points, or $T$ has a fixed point in each nonempty bounded closed convex set it leaves invariant. It is an open question whether every nonexpansive $T: C \rightarrow X$ satisfies (CFP) when $C$ is locally weakly compact.

Theorem 2. If $C$ is locally weakly compact, $T: C \rightarrow C$ is nonexpansive, and $T$ satisfies (CFP), then $F(T)$ is a nonexpansive retract of $C$. 
Remark. It is a consequence of the mean ergodic theorem that if $X$ is reflexive and $L: X \rightarrow X$ is linear with $\|L\| \leq 1$, then there exists a projection $P$ of $X$ onto $\operatorname{Ker}(I-L)$ with $\|P\| \leq 1$. Theorem 2 may be regarded as a nonlinear generalization of this fact.

Proof. Since the empty set is by definition a nonexpansive retract of $C$, we may assume $F(T) \neq \varnothing$.

Fix $z$ in $C$ and define $K=\{f(z): f \in N(F(T))\}$. Now $K$ is the image of $N(F(T))$ under the $z$ th coordinate projection map of $P$ onto $C_{z}$ (this projection is evaluation at $z$ ), and since $N(F(T)$ ) is compact (Lemma 1) and the projection continuous, $K$ must be weakly compact, hence bounded. Clearly $K$ is nonempty. If $f, g \in N(F(T))$ and $0 \leq \lambda \leq 1$ then $\lambda f+(1-\lambda) g$ is in $N(F(T))$, so $K$ is convex. Therefore $K$ is a bounded, nonempty, closed convex subset of $C$. Since $T \circ f \in N(F(T))$ whenever $f \in N(F(T))$, we also have $T(K) \subset K$.

But $T$ satisfies (CFP), has a fixed point, and leaves $K$ invariant; therefore $T$ has a fixed point in $K$. That is, there exists $h$ in $N(F(T))$ with $h(z)$ in $F(T)$.

Since this is so for each $z$ in $C$, by Theorem $1, F(T)$ is a nonexpansive retract of $C$. Q.E.D.

Corollary 1. Suppose $C$ is closed and convex, $T: C \rightarrow C$ is nonexpansive, $T$ satisfies (CFP), and the convex closure $\overline{c o} T(C)$ of the range of $T$ is locally weakly compact. Then $F(T)$ is a nonexpansive retract of $C$.

Proof. Again, we may suppose $F(T) \neq \varnothing$. Let $T_{0}$ be the restriction of $T$ to the set co $T(C)$. Then $T_{0}$ maps co $T(C)$ into itself, and it is readily verified that $T_{0}$ satisfies (CFP) on $\overline{c o} T(C)$. By Theorem 2 there exists a nonexpansive retraction $r$ of $\overline{c o} T(C)$ onto $F(T)$. One nonexpansive retraction of $C$ onto $F(T)$ is $r \circ T$. Q.E.D.

Example 1. The condition $T(C) \subset C$ in Theorem 2 is essential. We give an example of $T$ and $X$ where $T: C \rightarrow X$ is nonexpansive, $X$ and $X^{*}$ are uniformly convex, and $T$ satisfies (CFP), but $F(T)$ is not a nonexpansive retract of $C$.

Let $X$ be $R^{4}$ normed by $\left\|\left(x_{1}, x_{2}, x_{3}, x_{4}\right)\right\|=\left(\sum\left|x_{i}\right|^{4}\right)^{1 / 4}$. Let $e_{1}, e_{2}, e_{3}$, and $e_{4}$ be the usual unit vectors, and let $C$ be the linear span of the vectors $e_{1}+e_{2}$, $e_{2}+e_{3}$, and $e_{2}+e_{4}$. Let $T$ be the restriction to $C$ of the projection $\left(x_{1}, x_{2}, x_{3}, x_{4}\right)$ $1-\left(x_{1}, x_{2}, x_{3}, 0\right)$. Then $T$ is nonexpansive and $F(T)$ is the subspace spanned by $e_{1}+e_{2}$ and $e_{2}+e_{3}$. However, $F(T)$ is not a nonexpansive retract of $C$.

Suppose to the contrary that there does exist a nonexpansive retraction $f$ of $C$ onto $F(T)$. Fix $x$ in $C \backslash F(T)$ and set $z=x-f(x)$, so that $z \neq 0$. For any $v$ in $F(T)$ we have $f x-v \in F(T)$ since $F(T)$ is a linear subspace of $X$, so that $f(f x-v)=f x-v$. Since $f$ is nonexpansive we therefore have

$$
\|v\|=\|f x-(f x-v)\|=\|f x-f(f x-v)\| \leq\|x-(f x-v)\|=\|z+v\| .
$$


Thus

$$
\frac{1}{4}\|v\|^{4} \leq \frac{1}{4}\|z+v\|^{4} \quad \text { for all } v \text { in } F(T) .
$$

Since $F(T)$ is a subspace, we may take $t \neq 0$, substitute $t^{-1} v$ for $v$ in (3), multiply both sides of (3) by $t^{4}$, to obtain

$$
\frac{1}{4}\|v\|^{4} \leq \frac{1}{4}\|t z+v\|^{4}
$$

for all $v$ in $F(T)$ and $t \neq 0$-and obviously for $t=0$ as well. Thus the function $\phi(t)=\frac{1}{4}\|t z+v\|^{4}$ assumes a minimum at 0 . If $J$ denotes the gradient of $\frac{1}{4}\|\cdot\|^{4}$, this means $(J v, z)=0$ for all $v$ in $F(T)$. (Here $(J v, z)$ denotes the value of the linear functional $J v$ at the point $z$.) Therefore $z$ is annihilated by the linear span of $J(F(T))$.

Explicitly, $J\left(x_{1}, x_{2}, x_{3}, x_{4}\right)=\left(x_{1}^{3}, x_{2}^{3}, x_{3}^{3}, x_{4}^{3}\right)$, and it is readily calculated that $J\left(e_{1}\right), J\left(e_{2}\right)$, and $J\left(e_{3}\right)$ are contained in the linear span of $J(F(T))$. Therefore $z$ is annihilated by $J e_{i}$ for $i=1,2$, and 3 .

But the only vectors annihilated by $J e_{i}$ for $i=1,2$, and 3 are of the form $t e_{4}$ for some real $t$. Since $C$ does not contain any vectors of this form, $C$ does not contain $z$. This is a contradiction which means that $F(T)$ cannot be a nonexpansive retract of $C$.

Remark. We cannot repeal the assumption $T(C) \subset C$, but we can relax it. Felix Browder has communicated to the author a proof of the fact that if $C$ is bounded and $T: C \rightarrow X$ is Lipschitzian while $T$ maps the boundary $\partial C$ into $C$, then there exists $\lambda$ in $(0,1)$ such that $T_{\lambda}=\lambda T+(1-\lambda) I$ maps $C$ into $C$. Since $F\left(T_{\lambda}\right)=F(T)$ for $\lambda \neq 0$, this reduces theorems about fixed points of Lipschitzian mappings which send $\partial C$ into $C$ to theorems about mappings which send $C$ into $C$. It is not known whether $T_{\lambda}$ must satisfy (CFP) whenever $T$ does.

In a strictly convex space, the fixed-point set of a nonexpansive mapping is convex. This is true in a more general sense even if $X$ is not strictly convex:

Theorem 3. If $T: C \rightarrow C$ is nonexpansive, $T$ satisfies (CFP), and $\overline{\mathrm{co}} T(C)$ is locally weakly compact, then $F(T)$ is metrically convex.

Proof. A subset $F$ of $C$ is said to be metrically convex if for each pair of distinct points $x_{0}, x_{1}$ of $F$ there exists a point $\bar{x}$ of $F$, distinct from $x_{0}$ and $x_{1}$, such that $\left\|x_{0}-x_{1}\right\|=\left\|x_{0}-\bar{x}\right\|+\left\|\bar{x}-x_{1}\right\|$. By Corollary 1 it suffices to show that nonexpansive retracts of $C$ are metrically convex.

Let $r$ be a nonexpansive retraction of $C$ onto $F$, and let $x_{0}, x_{1}$ be two distinct points of $F$. For $t$ in $[0,1]$ let $x_{t}=(1-t) x_{0}+t x_{1}$ (so $x_{t} \in C$ ). Since $r\left(x_{0}\right)=x_{0}$ $\neq x_{1}=r\left(x_{1}\right)$ and $t \longmapsto r\left(x_{t}\right)$ is continuous, there exists $t$ in $(0,1)$ such that $r\left(x_{t}\right)$ is distinct from $x_{0}$ and $x_{1}$. Let $\bar{x}$ be one such $r\left(x_{t}\right)$. Then $\bar{x} \in F$ is distinct from $x_{0}$ and $x_{1}$, while

$$
\begin{aligned}
\left\|x_{0}-x_{1}\right\| & =\left\|r\left(x_{0}\right)-r\left(x_{1}\right)\right\| \leq\left\|r\left(x_{0}\right)-r\left(x_{t}\right)\right\|+\left\|r\left(x_{t}\right)-r\left(x_{1}\right)\right\| \\
& \leq\left\|x_{0}-x_{t}\right\|+\left\|x_{t}-x_{1}\right\|=\left\|x_{0}-x_{1}\right\|
\end{aligned}
$$


since $x_{0}, x_{t}$, and $x_{1}$ are collinear. Equality therefore holds throughout (5). In particular,

$$
\left\|r\left(x_{0}\right)-r\left(x_{1}\right)\right\|=\left\|r\left(x_{0}\right)-r\left(x_{t}\right)\right\|+\left\|r\left(x_{t}\right)-r\left(x_{1}\right)\right\| .
$$

But $r\left(x_{0}\right)=x_{0}, r\left(x_{1}\right)=x_{1}$, and $r\left(x_{t}\right)=\bar{x}$, hence

$$
\left\|x_{0}-x_{1}\right\|=\left\|x_{0}-\bar{x}\right\|+\left\|\bar{x}-x_{1}\right\| \text {. }
$$

Q.E.D.

Example 2. We use an example of De Marr [5] to show that the condition $T(C) \subset C$ is also essential in Theorem 3 .

Let $X$ be $R^{2}$ with the max norm, let $C$ be the line segment joining the two points $(1, \pm 1)$, and define $T: C \rightarrow X$ by $T(x, y)=(|y|, y)$. Then $T$ is nonexpansive and satisfies (CFP) (since $T$ is compact), but $F(T)$ consists of the two points $(1, \pm 1)$. Thus $F(T)$ is not metrically convex.

Now we turn to concrete conditions which guarantee that $T$ satisfies (CFP). First we introduce and recall some definitions used in the next theorem.

A set $K$ is said to have the fixed point property for nonexpansive mappings if every nonexpansive mapping of $K$ into $K$ has a fixed point in $K$; $C$ is said to have the hereditary fixed point property for nonexpansive mappings if every nonempty bounded closed convex subset of $C$ has the fixed point property for nonexpansive mappings. It is an open question whether the fixed point property implies the hereditary fixed point property.

We say that $X$ has compact faces if the only weakly compact convex subsets of the boundary of the unit ball are strongly compact. Obviously $X$ has compact faces if $X$ is strictly convex. Another sufficient condition for $X$ to have compact faces is that $X$ satisfy $\left(H_{L}\right)$ :

$$
x_{n} \rightarrow x \text { weakly and }\left\|x_{n}\right\| \rightarrow\|x\| \text { imply } x_{n} \rightarrow x \text { strongly. }
$$

A point $x_{0}$ of a bounded convex set $K$ is called a nondiametral point of $K$ if

$$
\sup _{y \in K}\left\|x_{0}-y\right\|<\operatorname{diameter} K .
$$

$C$ is said to have normal structure (Brodskii-Mil'man [3]) if each bounded closed convex subset $K$ of $C$ which contains more than one point contains a nondiametral point.

Theorem 4. Suppose $T: C \rightarrow X$ is nonexpansive. Then $T$ satisfies (CFP) if any one of the following is satisfied:

(a) $C$ has the hereditary fixed point property for nonexpansive mappings;

(b) $C$ is locally weakly compact and has normal structure;

(c) $C$ is locally weakly compact and $X$ has compact faces;

(d) $(I-T)(K)$ is strongly closed whenever $K$ is a bounded closed convex set;

(e) $T$ is compact;

(f) $T$ is affine and $C$ is locally weakly compact. 
Proofs. We may suppose that $T$ has a fixed point. Let $K$ be a nonempty bounded closed convex subset of $C$ which is invariant under $T$.

(a) The restriction of $T$ to $K$ is a nonexpansive mapping of $K$ into $K$, and since $C$ has the hereditary fixed point property, $T$ must have a fixed point in $K$. Q.E.D.

(b) We may rephrase the theorem of Kirk [7] as follows: if $C$ is locally weakly compact and has normal structure, then $C$ has the hereditary fixed point property for nonexpansive mappings. Hence (b) follows from (a). Q.E.D.

(c) We modify a proof that Edelstein [6, Proposition 3] gave for the case $X=R^{n}$. Fix $f_{0}$ in $F(T) . K$ is weakly compact, so the set $K_{0}=\left\{y \in K:\left\|f_{0}-y\right\|\right.$ $\left.=\operatorname{dis}\left(f_{0}, K\right)\right\}$ is nonempty. We also have $K_{0}=\left\{y \in K:\left\|f_{0}-y\right\| \leq \operatorname{dis}\left(f_{0}, K\right)\right\}$; hence $K_{0}$ is a weakly compact, convex subset of the boundary of a ball in $X$. Since $X$ is assumed to have compact faces, $K_{0}$ is compact.

However,

$$
\left\|T y-f_{0}\right\|=\left\|T y-T f_{0}\right\| \leq\left\|y-f_{0}\right\| \leq \operatorname{dis}\left(f_{0}, K\right)
$$

and $T y \in K$ whenever $y \in K_{0}$, so $T$ maps $K_{0}$ into $K_{0}$. By Schauder's theorem, $T$ has a fixed point in $K_{0}$. In particular, $T$ has a fixed point in $K$.

If $X$ is strictly convex (the condition used by Edelstein), we do not need Schauder's theorem. For, $K_{0}$ is a single point which must be a fixed point since $K_{0}$ is invariant under T. Q.E.D.

(d) Without loss of generality we may suppose $0 \in K$. Let $\left\{t_{n}\right\}$ be a sequence of real numbers drawn from $(0,1)$ such that $t_{n} \rightarrow 1$. Then $t_{n} T$ is a $t_{n}$-contraction and maps $K$ into $K$ since $K$ is convex and contains 0 . By the Banach contraction principle $t_{n} T$ has a fixed point $x_{n}$ in $K$. Since $x_{n}-T x_{n}=\left(1-t_{n}^{-1}\right) x_{n}$ and $t_{n} \rightarrow 1$ while $\left\{x_{n}\right\}$ is bounded, we have $x_{n}-T x_{n} \rightarrow 0$ strongly. Thus 0 lies in the closure of $(I-T)(K)$. By hypothesis this set is closed, so $0 \in(I-T)(K)$, i.e., $T$ has a fixed point in $K$. Q.E.D.

(e) If $T$ is compact, i.e. maps bounded sets into relatively strongly compact sets, then it is easy to show that $I-T$ maps closed bounded sets into closed sets. In particular, (e) follows from (d). Q.E.D.

(f) The image of a weakly compact, convex set under a continuous affine mapping is easily seen to be strongly closed. Therefore, if $K$ is a bounded closed convex subset of $C$, then $K$ is weakly compact, and since $I-T$ is affine, $(I-T)(K)$ is closed. Thus (f) follows from (d). Q.E.D.

3. Intersections and unions of nonexpansive retracts. Because of examples such as those already given, we have so far restricted ourselves to mappings which send $C$ into $C$. We have only one result in a more general direction:

Lemma 3. Suppose $X$ is strictly convex and $\left\{T_{n}\right\}$ is a sequence of nonexpansive mappings $T_{n}: C \rightarrow X$. Then there exists a nonexpansive mapping $T: C \rightarrow X$ such that $F(T)=\bigcap_{n} F\left(T_{n}\right)$. 
Proof. If the $T_{n}$ 's do not have a common fixed point we can let $T$ be translation by any nonzero vector; then both $F(T)$ and $\cap_{n} F\left(T_{n}\right)$ are empty.

Otherwise, let $y_{0}$ be a common fixed point of the $T_{n}$ 's. Let $\left\{\lambda_{n}\right\}$ be any sequence of positive real numbers such that $\sum_{n} \lambda_{n}=1$ and set $T=\sum_{n} \lambda_{n} T_{n}$. The mapping $T$ is well defined since

$$
\left\|T_{n} x\right\| \leq\left\|T_{n} x-T_{n} y_{0}\right\|+\left\|T_{n} y_{0}\right\| \leq\left\|x-y_{0}\right\|+\left\|y_{0}\right\|
$$

thus $\sum \lambda_{n} T_{n} x$ converges absolutely for each $x$ in $C$. Obviously $T$ is nonexpansive and maps $C$ into $X$.

We claim that $F(T)=\bigcap_{n} F\left(T_{n}\right)$. The inclusion $\bigcap_{n} F\left(T_{n}\right) \subset F(T)$ being trivial, we prove only the reverse inclusion.

Suppose, therefore, that $T x=x$. Then

$$
\begin{aligned}
\left\|x-y_{0}\right\| & =\left\|T x-y_{0}\right\|=\left\|\sum \lambda_{n} T_{n} x-y_{0}\right\| \\
& =\left\|\sum \lambda_{n}\left(T_{n} x-y_{0}\right)\right\| \leq \sum \lambda_{n}\left\|T_{n} x-y_{0}\right\| .
\end{aligned}
$$

But $T_{n} y_{0}=y_{0}$ and $T_{n}$ is nonexpansive, so $\left\|T_{n} x-y_{0}\right\| \leq\left\|x-y_{0}\right\|$. Since $\sum \lambda_{n}$ $=1,(6)$ implies

$$
\begin{aligned}
\left\|\sum \lambda_{n}\left(T_{n} x-y_{0}\right)\right\| & =\left\|x-y_{0}\right\| & & \text { and } \\
\left\|T_{n} x-y_{0}\right\| & =\left\|x-y_{0}\right\| & & \text { for all } n .
\end{aligned}
$$

Since $X$ is strictly convex and each $\lambda_{n}>0$ while $\sum \lambda_{n}=1$; (7) implies $T_{n} x-y_{0}$ $=T_{m} x-y_{0}$ for all $m, n$, i.e., $T_{n} x=T_{m} x$ for all $m, n$. Therefore

$$
x=T x=\sum_{n} \lambda_{n} T_{n} x=\sum_{n} \lambda_{n} T_{m} x=T_{m} x
$$

for all $m$. That is, $x \in \cap_{m} F\left(T_{m}\right)$. Q.E.D.

Remark. It is not known whether Lemma 3 is valid for uncountable families of nonexpansive mappings $T_{\alpha}: C \rightarrow X$. However:

Lemma 4. Suppose $C$ is locally weakly compact and $\left\{F_{\alpha}: \alpha \in A\right\}$ is a family of weakly closed nonexpansive retracts of $C$.

(a) If the family is directed by $\supset$, then $\bigcap_{\alpha} F_{\alpha}$ is a nonexpansive retract of $C$.

(b) If each $F_{\alpha}$ is convex and the family is directed by $\subset$, then $\mathrm{Cl} \cup_{\alpha} F_{\alpha}$ is a nonexpansive retract of $C$.

Proof of (a). Let $F=\cap_{\alpha} F_{\alpha}$. We may suppose $F \neq \varnothing$. Let $z \in C$ and define $K=\{f(z): f \in N(F)\}$. As in the proof of Theorem $2, K$ is weakly compact. Since $F_{\alpha}$ is weakly closed, $K \cap F_{\alpha}$ is weakly compact for all $\alpha$.

Furthermore, $K \cap F_{\alpha}$ is nonempty for each $\alpha$. Indeed, if $f_{\alpha}$ denotes a nonexpansive retraction of $C$ onto $F_{\alpha}$, then since $F \subset F_{\alpha}$ we have $f_{\alpha} \in N(F)$; hence $f_{\alpha}(z) \in K$. But $f_{\alpha}(z) \in F_{\alpha}$ since $f_{\alpha}$ is a retraction of $C$ onto $F_{\alpha}$. We have shown that $K \cap F_{\alpha} \neq \varnothing$.

Finally, since the $F_{\alpha}$ 's are directed by $\supset$, the family $\left\{K \cap F_{\alpha}: \alpha \in A\right\}$ has the finite intersection property. Since each $K \cap F_{\alpha}$ is weakly compact, the intersec- 
tion $\cap_{\alpha}\left(K \cap F_{\alpha}\right)$ is nonempty, so that $K \cap F \neq \varnothing$.

Hence there exists $h$ in $N(F)$ such that $h(z) \in F$. By Theorem $1, F$ is a nonexpansive retract of $C$. Q.E.D.

Proof of (b). Without loss of generality we may suppose there exists an $F_{0}$ in the family of $F_{\alpha}$ 's such that $F_{0} \subset F_{\alpha}$ for all $\alpha$.

Put $K=$ weak-cl $\cup_{\alpha} F_{\alpha}$. For each $\alpha \in A$ define $W_{\alpha}=\left\{f \in N\left(F_{0}\right): f(C)\right.$ $\subset K$ and $f x=x$ for all $x$ in $F_{\alpha}$ \}. Then $W_{\alpha} \neq \varnothing$ (it contains at least one nonexpansive retraction of $C$ onto $F_{\alpha}$ ).

First, $\left\{W_{\alpha}: \alpha \in A\right\}$ is directed by $\supset$. Indeed, if $\alpha, \beta \in A$ then there exists $\gamma \in A$ such that $F_{\alpha} \cup F_{\beta} \subset F_{\gamma}$ since the family is directed by $\subset$; clearly $W_{\gamma} \subset W_{\alpha} \cap W_{\beta}$.

In particular, $\left\{W_{\alpha}: \alpha \in A\right\}$ has the finite intersection property.

Second, it is readily seen that since $K$ is weakly closed, each $W_{\alpha}$ is closed in $N\left(F_{0}\right)$. Therefore $W_{\alpha}$ is compact.

It follows that there exists $f$ in $\cap_{\alpha} W_{\alpha}$. We claim that such an $f$ is a nonexpansive retraction of $C$ onto $K$.

$f$ is nonexpansive because $f$ is in $N(F) ; f(C) \subset K$ because $f$ is in each $W_{\alpha} . f$ fixes each point of $\cup_{\alpha} F_{\alpha}$ because $f$ is in each $W_{\alpha}$. By continuity, $f$ fixes each point of the strong closure of $\cup_{\alpha} F_{\alpha}$. But $\cup_{\alpha} F_{\alpha}$ is convex since the $F_{\alpha}$ 's are directed by $\subset$ and each $F_{\alpha}$ is convex. Therefore the strong closure of $\cup_{\alpha} F_{\alpha}$ is identical to $K$, the weak closure of $\cup_{\alpha} F_{\alpha}$.

We have shown that $f$ is nonexpansive, $f(C) \subset K$, and $K \subset F(f)$. But this implies that $f$ is a nonexpansive retraction of $C$ onto $K$. Q.E.D.

Theorem 5. Suppose $X$ is strictly convex, $C$ is locally weakly compact, and $\left\{T_{\alpha}: \alpha \in A\right\}$ is a family of nonexpansive mappings $T_{\alpha}: C \rightarrow C$. Then $\cap_{\alpha} F\left(T_{\alpha}\right)$ is a nonexpansive retract of $C$.

Proof. Without loss of generality we may suppose $\bigcap_{\alpha} F\left(T_{\alpha}\right)$ is nonempty. Let $\exists$ be the family of sets of the form $\cap\left\{F\left(T_{\lambda}\right): \lambda \in A^{\prime}\right\}$, where $A^{\prime}$ runs through all possible finite subsets of $A$. By Lemma 3 such an intersection is the fixed-point set of a nonexpansive $T: C \rightarrow X$. Such an intersection is nonempty since $\bigcap_{\alpha} F\left(T_{\alpha}\right) \neq \varnothing$, and the construction of $T$ in Lemma 3 shows that we may assume $T(C) \subset C$. By Theorem 2 and Theorem 4(c), $F(T)$ is a nonexpansive retract of $C . F(T)$ is convex because in a strictly convex space the fixed-point set of any nonexpansive mapping is convex.

Thus $\exists$ is a family of convex nonexpansive retracts of $C$ which are weakly closed because they are convex and strongly closed. Since $\Im$ is obviously directed by $\supset$, Lemma 4(a) implies that $\cap\{F: F \in \mathcal{Y}\}$ is a nonexpansive retract of $C$. But this is just $\bigcap_{\alpha} F\left(T_{\alpha}\right)$. Q.E.D.

Lemma 5. Suppose $X$ is reflexive and $F$ is a convex nonexpansive retract of $X$. Let $x_{0} \in F$ and define $K\left(x_{0}, F\right)=\mathrm{Cl} \cup_{t>0} t F+(1-t) x_{0}$. Then $K\left(x_{0}, F\right)$ is a nonexpansive retract of $X$. 
Remark. $K\left(x_{0}, F\right)$ is the cone with vertex $x_{0}$ generated by $F$. If $x_{0}$ belongs to the relative interior of $F$, then $K\left(x_{0}, F\right)$ is the smallest closed flat in $X$ which contains $F$. If $x_{0}$ is a boundary point of $F$ at which $F$ is smooth, $K\left(x_{0}, F\right)$ is the closed half-space which contains $F$ whose boundary is the tangent hyperplane to $F$ at $x_{0}$.

Proof of Lemma 5. For $t>0$ define $F_{t}=t F+(1-t) x_{0}$. If $f$ is a nonexpansive retraction of $X$ onto $F$, then

$$
x \longmapsto t \cdot f\left(t^{-1} x+\left(1-t^{-1}\right) x_{0}\right)+(1-t) x_{0}
$$

is a nonexpansive retraction of $X$ onto $F_{t}$; hence $F_{t}$ is a nonexpansive retract of $X$.

On the other hand, $F_{t}$ is obviously convex and $F_{s} \subset F_{t}$ when $0<s<t$. Since $X$ is reflexive, $X$ is locally weakly compact, so Lemma 4 (b) implies that $\mathrm{Cl} \cup_{t>0} F_{t}$ is a nonexpansive retract of $X$. Q.E.D.

Theorem 6. Suppose $X$ is strictly convex and reflexive. Let $\exists$ be the family of nonexpansive retracts of $X, \Im_{c}$ the family of cones in $X$ which are nonexpansive retracts of $X$. Then $\ni$ is closed under arbitrary intersections and $\vartheta_{c}, \cap$ generate $\ni$ (i.e., any set in $\exists$ is the intersection of some family of sets in $\exists_{c}$ ).

Proof. That $\Im$ is closed under arbitrary intersections follows immediately from Theorem 5, since a nonexpansive retract of $X$ is the fixed-point set of a nonexpansive mapping of $X$ into $X$.

In particular, the intersection of sets drawn from $\vartheta_{c}$ must be a nonexpansive retract of $X$. We have left to show only that every nonexpansive retract $F$ of $X$ is the intersection of a family of cones which are themselves nonexpansive retracts of $X$.

But $F$ is convex, being the fixed-point set of a nonexpansive mapping in a strictly convex space, and it easily follows from standard separation theorems that

$$
F=\bigcap\left\{K\left(x_{0}, F\right): x_{0} \in F\right\}
$$

By Lemma 5 , each $K\left(x_{0}, F\right)$ is a nonexpansive retract of $X$. Furthermore, each $K\left(x_{0}, F\right)$ is a cone in $X$ with vertex at $x_{0}$. Thus $F$ is the intersection of cones which are nonexpansive retracts of $X$. Q.E.D.

4. Common fixed points of commuting nonexpansive mappings. Belluce and Kirk [1] proved that if $C$ is weakly compact and has normal structure, then any finite family of commuting nonexpansive mappings $T_{\lambda}: C \rightarrow C$ has a common fixed point. We shall improve this by proving a result that is almost the best possible.

Lemma 6. Suppose $C$ is weakly compact and has the hereditary fixed point property for nonexpansive mappings. Suppose $F$ is a nonempty nonexpansive retract 
of $C$ and $T: C \rightarrow C$ is a nonexpansive mapping which leaves $F$ invariant. Then $F(T) \cap F$ is a nonempty nonexpansive retract of $C$.

Proof. Let $r$ be a nonexpansive retraction of $C$ onto $F$. Then $T \circ r: C \rightarrow C$ is nonexpansive and in fact maps $C$ into $F$ since $r(C) \subset F$ and $T$ leaves $F$ invariant. Since $C$ is bounded and has the hereditary fixed point property, $F(T r) \neq \varnothing$. By Theorem 2 and Theorem 4(a), $F(T r)$ is a nonexpansive retract of $C$.

Thus the lemma is proven once we show $F(T) \cap F=F(T r)$. The inclusion $\subset$ is trivial; to prove the reverse inclusion, suppose $\operatorname{Tr}(x)=x$. Since $r(x) \in F$ and $T$ leaves $F$ invariant, we therefore have $\operatorname{Tr}(x) \in F$, i.e. $x \in F$. But then $r(x)=x$, so that $\operatorname{Tr}(x)=x$ implies $T x=x$. That is, $x \in F(T) \cap F$. Q.E.D.

Theorem 7. Suppose $C$ is weakly compact and has the hereditary fixed point property for nonexpansive mappings. If $\left\{T_{j}: 1 \leq j \leq n\right\}$ is a finite family of commuting nonexpansive mappings $T_{j}: C \rightarrow C$ then $\bigcap_{j=1}^{n} F\left(T_{j}\right)$ is a nonempty nonexpansive retract of $C$. In particular, the set of common fixed points of the $T_{j}$ 's is connected.

Proof. By induction on $n$. The theorem is obviously true for $n=1$.

Suppose the theorem true for commuting families of a certain cardinality $n$, and suppose $\left\{T_{j}: 1 \leq j \leq n+1\right\}$ is a commuting family of cardinality $n+1$. Let $F=\bigcap_{j=2}^{n+1} F\left(T_{j}\right)$. By the induction hypothesis $F$ is a nonempty nonexpansive retract of $C$.

We claim that $F$ is invariant under $T_{1}$. In fact, if $x \in F$ then $T_{j} x=x$ for $j \geq 2$, so $T_{j} T_{1} x=T_{1} T_{j} x=T_{1} x$. That is, $T_{1} x \in F\left(T_{j}\right)$ for $j \geq 2$, so $T_{1} x \in F$.

By Lemma 6, $F\left(T_{1}\right) \cap F$ is a nonempty nonexpansive retract of $C$. But this set is just $\bigcap_{j=1}^{n+1} F\left(T_{j}\right)$. Q.E.D.

It is not known whether the conclusion of Theorem 7 is valid for infinite families. Belluce and Kirk [2] have extended their theorem of [1] to infinite families, at the expense of assuming that $C$ has "complete normal structure."

De Marr [5] proved the earliest of these theorems; we give an improved version of this result:

Theorem 8. Suppose $C$ is compact and $\left\{T_{\alpha}: \alpha \in A\right\}$ is a family of commuting nonexpansive mappings $T_{\alpha}: C \rightarrow C$. Then $\bigcap_{\alpha} F\left(T_{\alpha}\right)$ is a nonempty nonexpansive retract of $C$.

Proof. $C$ has the hereditary fixed point property for nonexpansive mappings, so Theorem 7 applies.

Let $\exists$ be the family of sets of the form $\cap\left\{F\left(T_{\lambda}\right): \lambda \in A^{\prime}\right\}$, where $A^{\prime}$ runs through all possible finite subsets of $A$. Such an intersection is compact since it is closed in the compact set $C$.

Thus, by Theorem $7, \Im$ consists of compact, nonempty nonexpansive retracts of $C$. Since $\exists$ is directed by $\supset$, Lemma $4($ a) implies that $\cap\{F: F \in \mathcal{Y}\}$ is a 
nonexpansive retract of $C$. This intersection is nonempty since each $F$ in $\exists$ is compact and $\Im$ has the finite intersection property.

The proof is concluded by noting that $\bigcap_{\alpha} F\left(T_{\alpha}\right)=\bigcap\{F: F \in \vartheta\}$. Q.E.D.

\section{REFERENCES}

1. L. P. Belluce and W. A. Kirk, Fixed-point theorems for families of contraction mappings, Pacific J. Math. 18 (1966), 213-217. MR 33 \# 7846.

2._, Nonexpansive mappings and fixed-points in Banach spaces, Illinois J. Math. 11 (1967), 474-479. MR 35 \# 5988.

3. M. S. Brodskiī and D. P. Mil'man, On the center of a convex set, Dokl. Akad. Nauk SSSR 59 (1948), 837-840. (Russian) MR 9, 448.

4. R. E. Bruck, Jr., Nonexpansive retracts of Banach spaces, Bull. Amer. Math. Soc. 76 (1970), 384-386. MR 41 \#794.

5. R. E. DeMarr, Common fixed-points for commuting contraction mappings, Pacific J. Math. 13 (1963), 1139-2241. MR 28 \#2446.

6. M. Edelstein, On nonexpansive mappings, Proc. Amer. Math. Soc. 15 (1964), 689-695. MR 29 \#2780

7. W. A. Kirk, A fixed point theorem for mappings which do not increase distances, Amer. Math. Monthly 72 (1965), 1004-1006. MR 32 \#6436.

Department of Mathematics, University of Southern California, los Angeles, CaliFORNIA 90007 